\title{
CENTRAL FUNCTIONS IN GROUP ALGEBRAS
}

\author{
RICHARD D. MOSAK
}

Abstract. In this paper we show that for a locally compact group $G$, the group algebra $L_{1}(G)$ has nontrivial center if and only if $G$ possesses a compact neighborhood of 1 , invariant under inner automorphisms. Moreover, $G$ has a basis of such neighborhoods at 1 if and only if $L_{1}(G)$ has an approximate identity consisting of functions in the center of $L_{1}$. This constitutes part of a program of finding conditions on the group algebra which characterize groups satisfying various compactness conditions (see e.g., [3]).

Godement remarks in $[1$, p. $28 \mathrm{n}$ and Théorème 6$]$ that for a locally compact unimodular group $G$, the existence of a nontrivial function in $L_{2}(G)$ invariant under inner automorphisms implies the existence of a compact neighborhood of 1 in $G$, invariant under inner automorphisms. We show in this note that Godement's method can be extended to deal with similar conditions on $L_{1}(G)$; we make use of an earlier observation to consider more generally the case of nonunimodular groups as well. Our result is a characterization by conditions on the group algebra of the classes [IN] and [SIN] of locally compact groups (as in [2], we say that $G$ is an [IN] group if $G$ contains a compact invariant neighborhood of the identity, and an [SIN] group if it contains a fundamental family of compact invariant neighborhoods of the identity).

This note thus constitutes part of a program of finding conditions on the function-space level to characterize classes of locally compact groups satisfying certain compactness conditions. For this program results on the $L_{1}$ level appear to be more natural than those for $L_{2}$ functions or continuous functions with compact support. In this respect our note strengthens the existing result of Godement mentioned above, and that of [2, Proposition (2.6)] concerning a related condition for continuous functions. (For another result in the general program, see $[3$, Theorem (3.1)], which gives necessary and sufficient conditions on $L_{1}(G)$ for $G$ to be an [MAP] group.) We might remark that the direct part of (b), in our proposition, is used repeatedly in the study of harmonic analysis on [Z] groups and [FIA]- groups; see $[3,(6.18)]$ and $[5]$.

Received by the editors October 15, 1970.

AMS 1970 subject classifications. Primary 22D15, 22D05, 43A20.

Key words and phrases. Locally compact group, group algebra, convolution, center of an algebra, central function, approximate identity, compact invariant neighborhood of 1 . 
Throughout the paper we shall let $d y$ denote left Haar measure on $G$, and let $\Delta$ denote the modular function. We use "invariant" to mean "invariant under inner automorphisms," and "central $L_{1}$ function" to mean "function contained in the center of the algebra $L_{1}(G)$." In particular, we shall say that an $L_{1}$ function is invariant iff for each $t$ in $G, f\left(t x t^{-1}\right)=f(x)$ for almost all $x$, where the null set may depend on $t$.

Proposition. Let $G$ be a locally compact group.

(a) $G$ is an [IN] group iff $L_{1}(G)$ has a nontrivial center.

(b) $G$ is an [SIN] group iff $L_{1}(G)$ has an approximate identity consisting of central functions.

Proof of (a). We use the fact (see Proposition 1.2 of [5]) that an $L_{1}$ function $f$ is central iff $f$ satisfies the following condition: for each $t$ in $G, f\left(t x t^{-1}\right)=f(x) \Delta(t)$ a.e. $x$, where the null set may depend on $t$. Thus, as is well known, for a unimodular group the central elements in $L_{1}(G)$ consist of the invariant $L_{1}$ functions. If $G$ has a compact invariant neighborhood $V$ of 1 , then $G$ is unimodular; hence the characteristic function of $V$ is central. Suppose conversely that $f$ is a nontrivial central $L_{1}$ function. If $h=|f|^{1 / 2}$, then $h$ is a positive $L_{2}$ function, and $h\left(t x t^{-1}\right)=\Delta^{1 / 2}(t) h(x)$ a.e. $x$. Let

$$
g(s)=h * h^{\sim}(s)=\int_{G} h(s y) h(y) d y \quad\left(\text { where } k^{\vee}(y)=\overline{k\left(y^{-1}\right)}\right) ;
$$

then $g$ is a continuous function vanishing at infinity $[4,20.16]$.

Also, $g$ is invariant under inner automorphisms: for if $s$ and $t$ are fixed, then

$$
g\left(t s t^{-1}\right)=\int h\left(t s t^{-1} y\right) h(y) d y=\Delta(t)^{-1} \int h\left(t s y t^{-1}\right) h\left(t y t^{-1}\right) d y .
$$

But except on a null set in $y$ depending only on $s$ and $t$, we have $h\left(t s y t^{-1}\right)=\Delta^{1 / 2}(t) h(s y)$ and $h\left(t y t^{-1}\right)=\Delta^{1 / 2}(t) h(y)$, so

$$
g\left(t s t^{-1}\right)=\Delta^{-1}(t) \Delta^{1 / 2}(t) \Delta^{1 / 2}(t) \int h(s y) h(y) d y=g(s) .
$$

Finally, $g(1)=\|h\|_{2}^{2}=\|f\|_{1}>0$. Therefore, for any $\epsilon$ such that $g(1)$ $>\epsilon>0$, the set $\{x \in G:|g(x)| \geqq \epsilon\}$ is a compact invariant neighborhood of 1 .

Proof of (b). It is clear that if $G$ has a fundamental system of compact invariant neighborhoods of 1 , then the (suitably normalized) characteristic functions of these neighborhoods form a central approximate identity for $L_{1}(G)$. Conversely, suppose $L_{1}(G)$ has a 
central approximate identity $\left\{f_{\alpha}\right\}_{\alpha \in A}$, and let $x \in G, x \neq 1$. Note that (as in (a)) $G$ is unimodular; hence the central functions are invariant. We observe first that the operator on $L_{1}(G)$ of left translation by $x$ (which we denote by $L_{x}$ ) cannot reduce to the identity on $\left\{f_{\alpha}\right\}$. For if $L_{x}\left(f_{\alpha}\right)=f_{\alpha}$ for all $\alpha$, then for any $k$ in $L_{1}(G)$ we have $L_{x}\left(f_{\alpha}\right) * k=f_{\alpha} * k$; hence (since left translations commute with right translations and therefore right convolutions) $L_{x}\left(f_{\alpha} * k\right)=f_{\alpha} * k$. Since by hypothesis $\left\{f_{\alpha} * k: k \in L_{1}(G), \alpha \in A\right\}$ is dense in $L_{1}(G)$, this would imply that $L_{x}(f)=f$ for all $f \in L_{1}(G)$, which contradicts $x \neq 1$ (take $f$ to be the characteristic function of a compact neighborhood $U$ of 1 , with $x \notin U)$. Thus there is a nonzero central function $f_{\alpha} \in L_{1}(G)$ such that $L_{x}\left(f_{\alpha}\right) \neq f_{\alpha}$. Now, $f_{\alpha}=\left(f_{1}-f_{2}\right)+i\left(f_{3}-f_{4}\right)$, where the $L_{1}$ functions $f_{1}, f_{2}$, $f_{3}, f_{4}$ are real-valued and positive. Moreover, $f_{i}$ is central for each $i=1,2,3,4$; for it is clear that the invariance of $f_{\alpha}$ implies the invariance of its real and imaginary parts, and then of its positive and negative parts. Thus finally there is a nonzero positive central function $f \in L_{1}$ such that $L_{x} f \neq f$.

As in the proof of (a), set $h=f^{1 / 2}$, and $g(s)=h * h^{2}(s)=\left(h, L_{s} h\right)$ where we again denote by $L_{s}$ left translation in $L_{2}(G)$. Then evidently $L_{x} h \neq h$, and following Godement, we observe that $|g(x)| \neq g(1)$. For by the Cauchy-Schwarz inequality we have $|g(x)|=\left|\left(h, L_{x} h\right)\right|$ $\leqq\|h\|_{2}^{2}=g(1)>0$; if in addition $|g(x)|=g(1)$, then by the limiting case of that inequality we must have $L_{x} h=c h$ for some scalar $c>0$ (since $h$ and $L_{x} h$ are positive). But then $|g(x)|=|(h, c h)|=c(h, h)=c g(1)$, so that $c=1$, which contradicts the fact that $L_{x} h \neq h$. Now let

$$
V=\{S \in G:|g(s)| \geqq(|g(x)|+g(1)) / 2\} .
$$

Since as above $g$ is continuous, invariant, and vanishes at infinity, $V$ is a compact invariant neighborhood of 1 not containing $x$. Hence the family of all compact invariant neighborhoods of 1 has trivial intersection, and since this family is closed under finite intersections, a compactness argument (see [1, Théorème 6]) completes the proof.

Since any closed subgroup of an [IN] group is again an [IN] group, we have the following

CoRollary. Let $G$ be a locally compact group, and $H$ a closed subgroup. If $L_{1}(G)$ has a nontrivial center, then $L_{1}(H)$ has a nontrivial center.

Note that here $L_{1}(H)$ is the set of (equivalence classes of) integrable functions on $H$ with respect to left Haar measure on $H$. In general, if $H$ is not open, merely restricting a central function $f$ in $L_{1}(G)$ to $H$ 
will not give an $L_{1}$ function on $H$, while restricting the measure $f \cdot d x$ to $H$ will give the zero measure.

\section{BIBLIOGRAPHY}

1. R. Godement, Mémoire sur la theorie des caractères dans les groupes localement compacts unimodulaires, J. Math. Pures Appl. (9) 30 (1951), 1-110. MR 13, 12.

2. S. Grosser and M. Moskowitz, Compactness conditions in topological groups, I, II, J. Reine Angew. Math. (to appear).

3. - Harmonic analysis on central topological groups, Trans. Amer. Math. Soc. 26 (1971), 419-454.

4. E. Hewitt and K. Ross, Abstract harmonic analysis. Vol. I: Structure of topological groups. Integration theory, group representations, Die Grundlehren der math. Wissenschaften, Band 115, Academic Press, New York; Springer-Verlag, Berlin, 1963. MR 28 \#158.

5. R. Mosak, The $L^{1}$ - and $C^{*}$-algebras of $[\mathrm{FIA}]_{\bar{B}}^{-}$groups, and their representations (to appear).

Yale University, New Haven, Connecticut 06520 